\title{
Hepatic adenomas with synchronous or metachronous fibrolamellar carcinomas: both are characterized by LFABP loss
}

\author{
Rondell P Graham ${ }^{1}$, Luigi M Terracciano ${ }^{2}$, Alexander Meves ${ }^{3}$, Patrick M Vanderboom ${ }^{4}$, \\ Surendra Dasari ${ }^{5}$, Matthew M Yeh ${ }^{6}$, Michael S Torbenson ${ }^{1}$ and Michael W Cruise ${ }^{7}$ \\ ${ }^{1}$ Division of Anatomic Pathology, Department of Pathology, Mayo Clinic, Rochester, MN, USA; ${ }^{2}$ Institute of \\ Pathology, University Hospital Basel, Basel, Switzerland; ${ }^{3}$ Department of Dermatology, Mayo Clinic, \\ Rochester, MN, USA; ${ }^{4}$ Proteomics Core, Medical Genome Facility, Mayo Clinic, Rochester, MN, USA; \\ ${ }^{5}$ Department of Health Sciences Research, Mayo Clinic, Rochester, MN, USA; ${ }^{6}$ Department of Anatomic \\ Pathology, University of Washington Medical Center, Seattle, WA, USA and ${ }^{7}$ Department of Anatomic \\ Pathology, Cleveland Clinic, Cleveland, OH, USA
}

\begin{abstract}
Rare hepatic adenomas are associated with synchronous or metachronous fibrolamellar carcinomas. The morphology of these adenomas has not been well described and they have not been subclassifed using the current molecular classification schema. We examined four hepatic adenomas co-occurring with or preceding a diagnosis of fibrolamellar carcinoma in three patients. On histological examination, three of the adenomas showed the typical morphology of HNF1-a inactivated adenomas, whereas one showed a myxoid adenoma morphology. All of the adenomas were negative for PRKACA rearrangements by Fluorescence in situ Hybridization (FISH) analysis. All four of the adenomas showed complete loss or significant reduction of liver fatty acid binding protein (LFABP) expression by immunohistochemistry. Interestingly, the fibrolamellar carcinomas in each case also showed loss of LFABP by immunohistochemistry. One of the fibrolamellar carcinomas was negative for PRKACA rearrangements by FISH, whereas the others were positive. To investigate if LFBAP loss is typical of fibrolamellar carcinomas in general, an additional cohort of tumors was studied $(n=19)$. All 19 fibrolamellar carcinomas showed the expected PRKACA rearrangements and immunostains showed loss of LFABP in each case, consistent with HNF1- $a$ inactivation. To validate this observation, mass spectrometry-based proteomics was performed on tumor-normal pairs of six fibrolamellar carcinomas and showed an average 10-fold reduction in LFABP protein levels, compared with matched normal liver tissue. In conclusion, hepatic adenomas co-occurring with fibrolamellar carcinomas show LFABP loss and are negative for PRKACA rearrangements, indicating they are genetically distinct lesions. These data also demonstrate that LFABP loss, which characterizes HNF1- $a$ inactivation, is a consistent feature of fibrolamellar carcinoma, indicating HNF1- $a$ inactivation is an important event in fibrolamellar carcinoma pathogenesis.
\end{abstract}

Modern Pathology (2016) 29, 607-615; doi:10.1038/modpathol.2016.59; published online 25 March 2016

Fibrolamellar carcinoma is a rare subtype of primary liver carcinoma that affects younger patients without underlying chronic liver disease. The carcinoma is histologically distinct and shows a consistent somatic deletion leading to DNAJB1-PRKACA fusion transcripts. ${ }^{1,2}$ Although the tumorigenic mechanism for this fusion protein is not yet proven, the fusion

Correspondence: Dr MS Torbenson, MD, Division of Anatomic Pathology, Department of Pathology, Mayo Clinic, 200 First Street Southwest, Rochester, MN 55905, USA.

E-mail: Torbenson.michael@mayo.edu

Received 12 November 2015; revised 11 February 2016; accepted 11 February 2016; published online 25 March 2016 protein appears to drive overexpression of PRKACA. PRKACA is one of the catalytic subunits of protein kinase A, a key regulatory kinase with numerous downstream signaling targets.

Rarely, hepatic adenomas can co-occur with fibrolamellar carcinomas, either as synchronous or metachronous tumors. Although documented in a case report ${ }^{3}$ and a review article, ${ }^{4}$ these adenomas have not been fully described at the histological level and have not been subclassified using the current immunohistochemical panel of liver fatty acid binding protein (LFABP), C-reactive protein (CRP), serum amyloid A (SAA), beta catenin, and glutamine synthesis. In addition, questions persist as 
to whether these adenomas are simply misdiagnosed fibrolamellar carcinomas, misdiagnosed because of solid growth patterns with limited intratumoral fibrosis, an important diagnostic pitfall in the pathology of fibrolamellar carcinomas. ${ }^{4}$ The initial goals of this study were to describe the morphology of these adenomas, subtype them using the current hepatic adenoma classification system, and use molecular methods to determine if these adenomas share the distinctive DNAJB1-PRKACA fusion transcripts of fibrolamellar carcinoma. Based on our findings, we extended the study to determine if LFABP loss was characteristic of fibrolamellar carcinomas.

\section{Materials and methods}

We identified hepatic adenomas co-occurring with fibrolamellar carcinomas by searching the consultation or institutional files of several of the authors (LMT, MST, and MWC). Representative slides were centrally reviewed (RPG and MST). Formalin-fixed paraffin-embedded tissue blocks were chosen for immunohistochemistry, mass spectrometry-based proteomics, and molecular cytogenetic studies. Based on the initial study findings, additional material from 19 fibrolamellar carcinomas was selected for LFABP testing, including a group of 11 fibrolamellar carcinomas previously shown to harbor DNAJB1-PRKACA ${ }^{1}$ and an additional cohort of 8 fibrolamellar carcinomas.

\section{Immunohistochemistry}

Five micron-thick sections were cut from the tissue block and immunohistochemistry performed using antibodies to Arginase-1(predilute, clone SP156; Cell Marque), HepPar1 (predilute, clone OCH1E5; Ventana), cytokeratin 7 (1:100, Clone OV-TL 12/30; Dako), CD68 (clone KP1, 1:50-1:100; Dako), PRKAR1A (1:2000, clone OTI6C7; Origene Technologies), LFABP (1:50, rabbit polyclonal; Abcam), CRP (1:2000, clone Y284; Abcam), SAA (1:2000, clone mc1; Dako), Glutamine synthetase (1:2000, clone GS-6; Millipore), and beta catenin (predilute, clone 14; Ventana). Steam antigen retrieval and standard clinical methods were used for each antibody.

\section{Fluorescence in situ Hybridization (FISH)}

FISH for PRKACA rearrangement was performed on $5 \mu$-thick formal-fixed sections. PRKACA FISH was performed on the hepatic adenomas and the paired fibrolamellar carcinomas. In addition, we examined the untested fibrolamellar carcinomas in the followup cohort $(n=8)$. PRKACA FISH used the validated probe and methodology as described previously. ${ }^{1}$ FISH slides were visualized and scored by two experienced technologists who each independently evaluated 50 tumor nuclei.

\section{Mass Spectrometry-based Proteomic Analysis}

On six randomly selected fibrolamellar carcinomas, ${ }^{1}$ known to harbor the DNAJB1-PRKACA fusion transcript, we performed laser capture microdissection of areas of tumor and paired normal liver tissue on $10-\mu$ tissue sections cut on to PEN membrane slides. Approximately an $500000 \mu^{2}$ area of tissue was collected for each sample by laser capture. Collected fragments were captured into a tube containing lysis buffer, composed of $0.1 \mathrm{M}$ Tris-HCl $(\mathrm{pH}=8.5)$ with $0.002 \%$ Zwittergent Z3-16, via laser pressure catapulting. Proteins were extracted from the tissue fragments by heating the mixture at $99^{\circ} \mathrm{C}$ for $1 \mathrm{~h}$. Protein concentration of each microdissection was interpolated from a concentration vs area curve, which was pre-constructed by collecting different areas on a control tissue and estimating the resulting protein concentration with a BCA assay (ThermoFisher Scientific).

Proteins present in the patient samples were reduced by incubating at $56^{\circ} \mathrm{C}$ with $2.5 \mu \mathrm{l}$ of Tris in $0.1 \mathrm{M}$ Tris-HCl $(\mathrm{pH}=8.5$; final concentration $=10 \mathrm{mM})$ for $30 \mathrm{~min}$. Reduced proteins were alkylated by adding $2.5 \mu \mathrm{l}$ of iodoacetamide in $0.1 \mathrm{M}$ Tris- $\mathrm{HCl}(\mathrm{pH}=8.5$; final concentration of $10 \mathrm{mM}$ ) and incubating in the dark at room temperature for $30 \mathrm{~min}$. Proteins were digested by adding $2 \mu \mathrm{l}$ of trypsin to an enzyme to protein ratio of $1: 50$ and incubating at $37^{\circ} \mathrm{C}$ for $16 \mathrm{~h}$. The digestion was terminated by adding $3 \mu \mathrm{l}$ of $0.2 \%$ triflouroacetic acid (TFA) and the samples were diluted to a protein content of $12.5 \mathrm{\eta g} / \mathrm{\mu l}$ with a solution containing $0.2 \%$ formic acid (FA), $0.1 \%$ TFA, and.002\% Z3-16.

Peptides present in each sample were loaded onto a $0.25 \mu \mathrm{l}$ bed OptiPak tap (Optimize Technologies) custom-packed with $5 \mu \mathrm{m}$, 200A Magic C8 (BrukerMichrom) stationary phase. The loaded trap was washed for $4 \mathrm{~min}$ with an aqueous loading buffer of $0.2 \% \mathrm{FA}$ and $0.05 \% \mathrm{TFA}$ at $10 \mu \mathrm{l} / \mathrm{min}$. Following the wash, peptides were transferred onto a $35 \mathrm{~cm} \times$ $100 \mu \mathrm{m}$ PicoFrit column, self-packed with Agilent Poroshell 120S $2.7 \mu \mathrm{m}$ EC-C18 stationary phase, using a Dionex UltiMate 3000 RSLC liquid chromatography (LC) system (Thermo-Fisher Scientific). Peptides were separated using a $400 \mathrm{nl} / \mathrm{min} \mathrm{LC}$ gradient comprised of $2-40 \% \mathrm{~B}$ in $0-120 \mathrm{~min}$, $40-95 \%$ B from $120-130 \mathrm{~min}, 95 \% \mathrm{~B}$ from $130-$ $140 \mathrm{~min}$, and re-equilibrated to $2 \% \mathrm{~B}$ from $140-$ 150 min. The mobile phase A was $2 \%$ acetonitrile (ACN) in water with $0.2 \% \mathrm{FA}$ and the mobile phase B was ACN/isopropanol/water (80/10/10 by volume) with $0.2 \%$ FA. Eluting peptides were analyzed using a QExactive Plus mass spectrometer (Thermo-Fisher Scientific). The instrument was configured to operate in data-dependent mode by collecting MS1 data at 70000 resolving power (measured at $\mathrm{m} / \mathrm{z} 200$ ) with an AGC value of 1E6 over a $\mathrm{m} / \mathrm{z}$ range of 360-2000, using lock masses from background polysiloxanes at $\mathrm{m} / \mathrm{z} 371.10123$ and 446.12002. Precursors were fragmented with normalized collision 
energy of 28, fragments measured at 17500 resolving power and a fixed first mass of 140. Resulting tandem mass spectra (MS/MS) were collected on the top 20 precursor masses present in each MS1 using an AGC value of 1E5, max ion fill time of 50ms, and an isolation window of 1.5 Da.

\section{Bioinformatics}

The number of MS/MS matching to a protein in a sample is considered to be a semi-quantitative measure of its abundance. ${ }^{5}$ We have successfully used this method to discover biomarkers for detecting amyloid deposits present in fat aspirate specimens $^{6}$ and use this method for subtyping amyloid deposits present in clinical specimens. ${ }^{7}$ We utilized the same method for detecting differentially expressed proteins between fibrolamellar carcinoma and paired normal liver. ${ }^{8}$

To accomplish this, the MyriMatch database search engine was used to match the MS/MS present in data files against a composite protein sequence database containing RefSeq human proteome (version 53) and sequences common contaminants (eg wool and cotton). Reversed protein sequences were appended to the database for estimating peptide and protein false discovery rates. MyriMatch was configured to use $10 \mathrm{ppm} \mathrm{m/z} \mathrm{tolerance} \mathrm{for} \mathrm{precursors}$ and fragments while performing peptide-spectrum matching (PSM). The software derived semitryptic peptides from the sequence database while looking for the following variable modifications: carbamidomethylation of cysteine $(+57.023 \mathrm{Da})$, oxidation of methionine (+15.994 Da), and formation of n-terminal pyroglutamic acid (-17.023 Da). IDPicker software filtered the PSMs at $2 \%$ FDR. The software was configured to use an optimal combination of $\mathrm{MVH}$, mzFidelity, and XCorr scores for filtering. ${ }^{9}$ Protein identifications with at least two unique peptide identifications were considered to be present in the sample. Resulting proteins were clustered into groups of proteins that match the same set of peptides. QuasiTel software performed differential expression of protein groups found in two different experimental groups using a quasi-likelihood-based generalized linear mixture model. Protein groups with a $P$-value $\leq 0.05$ and an absolute fold change $\geq 1$ (where 0.0 signifies no change) were considered as significantly differentially expressed between fibrolamellar carcinoma and matched normal liver.

\section{Results}

\section{Gross and Microscopic Findings}

Four cases of hepatic adenomas with fibrolamellar carcinomas were identified in three patients (Table 1). In each case, the hepatic adenomas were separate lesions from the fibrolamellar carcinomas on both gross examination and microscopic examination. No transition zones were seen.

The first set of three adenomas occurring in two individuals are discussed first, because the adenomas had the same morphology. One individual had both a primary resection for fibrolamellar carcinoma and a subsequent resection for recurrent disease: each resection specimen showed a single adenoma in addition to the fibrolamellar carcinoma, with the adenomas measuring $2.7 \mathrm{~cm}$ in the primary resection and $0.5 \mathrm{~cm}$ in the resection for recurrent fibrolamellar carcinoma. A second individual had hepatic adenomatosis, with $>10$ adenomas. In all of these resection specimens, the hepatic adenomas were distinctly separate nodules from the fibrolamellar carcinoma, though they were adjacent to the fibrolamellar carcinoma in two specimens. Both individuals were women, with an average age of 27 years. All three hepatic adenomas were morphologically similar, with histological findings typical of $H N F 1-\alpha$ inactivated adenomas (Figure 1a). They all showed macrovesicular steatosis, from 10 to $70 \%$, with no balloon cells, no significant intratumoral inflammation, and no intratumoral fibrosis. In the hepatic adenomatosis case, all of the adenomas showed similar features. None of the adenomas showed cytological atypia. None had the cytological features that typify fibrolamellar carcinoma: big pink cells, prominent nucleoli, and intratumoral fibrosis. In contrast, the co-occurring fibrolamellar carcinomas all showed the classic features of large eosinophilic cells with granular cytoplasm, prominent nucleoli, and abundant intratumoral fibrosis (Figure 1b). The fibrolamellar carcinomas had no other unusual histological features. They ranged in size from 7.4 to $8.5 \mathrm{~cm}$.

The fourth adenoma case was resected in a 14-year-old female with a maternal history of the Carney complex and was previously reported as part of an array comparative genomic hybridization study. ${ }^{3}$ In this case, the adenoma measured $10 \mathrm{~cm}$ and was characterized by the recently described myxoid morphology (Figure 1c). ${ }^{10}$ The tumor

Table 1 Summary of index cases

\begin{tabular}{llllll}
\hline Index patient & Gender & Temporal relationship of tumors & $\begin{array}{l}\text { Hepatic adenoma } \\
\text { size (cm) }\end{array}$ & Adenomatosis & FLC size (cm) \\
\hline First & $\mathrm{F}$ & Synchronous & Primary resection, 2.7 & $\mathrm{N}$ & Primary, 8 \\
& & & Recurrence resection, 0.6 & $\mathrm{Y}$ & 1 \\
Second & $\mathrm{F}$ & Synchronous & Adenomatosis & $\mathrm{Y}$ & 8.5 \\
Third & $\mathrm{F}$ & Metachronous & 10 & $\mathrm{~N}$ & 7.4 \\
\hline
\end{tabular}




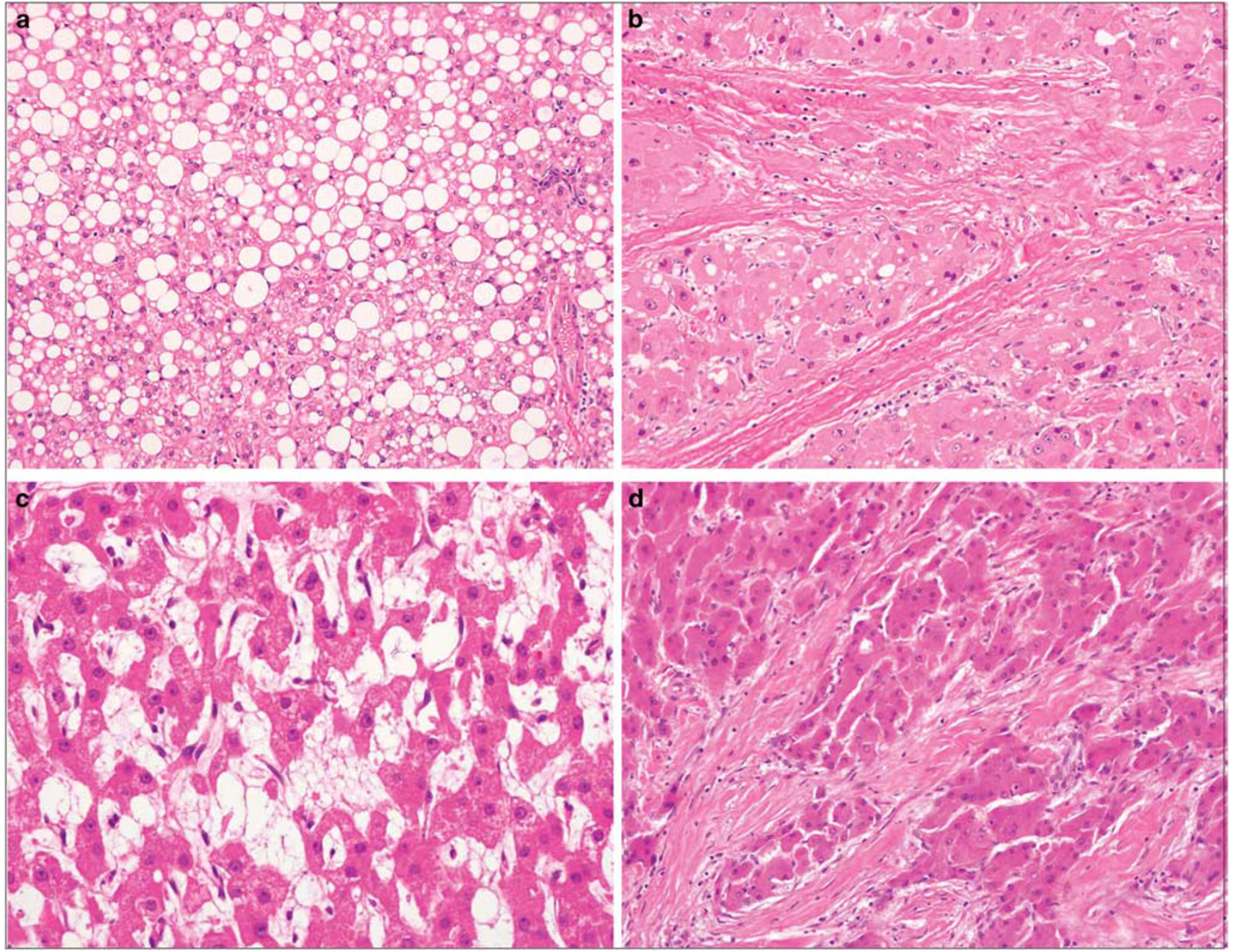

Figure 1 Morphological findings in livers with hepatic adenomas and fibrolamellar carcinomas. (a) The hepatic adenomas in the first three cases showed bland tumor cells with mild fatty change, findings typical of HNF1 alpha inactivated adenomas. (b) The co-existing fibrolamellar carcinomas showed the classical histological findings, with no unusual features. (c) In the fourth case, the adenoma showed a myxoid morphology. (d) The fibrolamellar carcinoma in the fourth case was also histologically typical.

showed cords and trabeculae of cytologically bland tumor cells widely separated by abundant extracellular myxoid material. Five years after the adenoma was resected, a fibrolamellar carcinoma developed in the same lobe of the liver. The resected fibrolamellar carcinoma showed classic histological features, with no unusual findings (Figure 1d).

\section{Immunohistochemical and Molecular Findings}

All four hepatic adenomas were negative for PRKACA rearrangements by FISH. The three synchronous adenomas were all classified as type 1 hepatic adenomas, or HNF1- $\alpha$ inactivated adenomas, by immunohistochemistry. They all showed complete of loss of LFABP staining (Figure 2a), with negative or patchy weak staining for CRP and SAA, and membranous beta catenin labeling without nuclear accumulation. Glutamine synthetase did not show strong diffuse staining in any of the cases.
In contrast to these findings, the adenoma in the patient whose mother had the Carney complex demonstrated partial loss of LFABP (Figure 2b) as well as partial positivity for CRP (Figure 2c), and normal results for the beta catenin, SAA, and glutamine synthetase. This adenoma was thus classified as type 4 in the current classification system (unclassifiable).

Immunostains were also performed on the fibrolamellar carcinomas. All were positive for either Arginase-1 $(N=3)$ or HepPar $1(N=1)$ and were diffusely and strongly cytokeratin 7 and CD68 positive $(N=4)$. For additional studies, the three primary fibrolamellar carcinomas were tested, but not the fibrolamellar carcinoma recurrence of the first case. In each of these three cases, the expression of LFABP was markedly diminished or absent (Figure 2d). All three were negative for nuclear accumulation of beta catenin and all showed patchy weak expression of SAA and were positive for CRP. By FISH, two of the three fibrolamellar carcinomas 


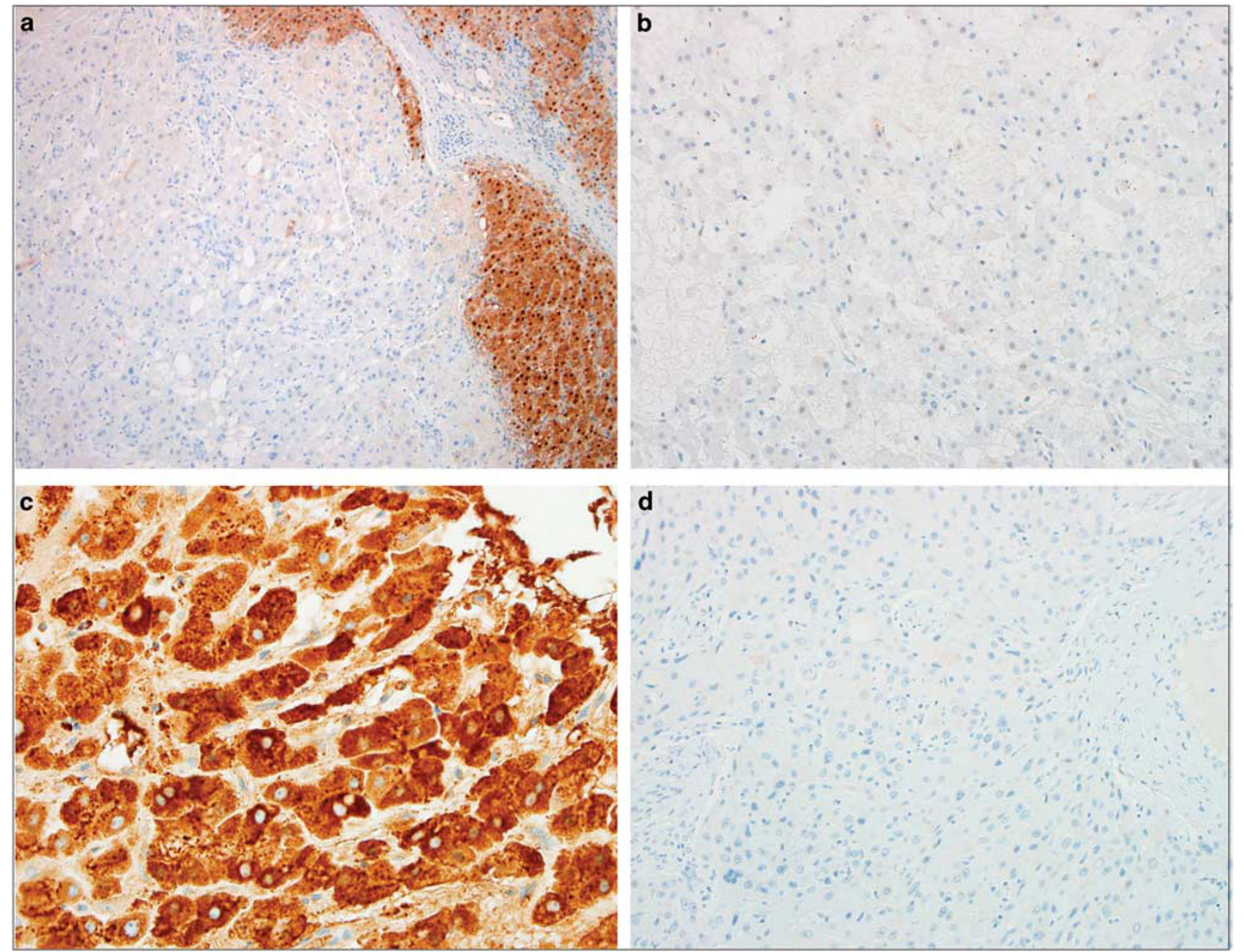

Figure 2 Immunostains in adenomas and fibrolamellar carcinomas. (a) The hepatic adenomas in case one although all three showed complete loss of LFABP staining. (b) In the fourth case, LFABP staining showed patchy loss. (c) The fourth case also showed patchy C-reactive protein (CRP) staining. (d) The fibrolamellar carcinomas in each of the four cases showed loss of LFABP staining.

harbored PRKACA rearrangements within all of the neoplastic cells of the (Figure 3a). However, the fibrolamellar carcinoma arising in the individual with a family history of the Carney Complex did not harbor PRKACA rearrangements by FISH (Figure $3 \mathrm{~b}$ ). RT-PCR was then performed and again showed no PRKACA rearrangements. However, re-review of the H\&E stains confirmed that the morphological findings were typical of fibrolamellar carcinoma and the diagnosis was further confirmed by co-expression of CK7 and CD68. ${ }^{11}$

Of note, the history of the Carney complex strongly suggests a germline mutation in PRKAR1A, which could represent an alternative mechanism of abnormal activation of protein kinase A. To follow-up on this possibility, we studied the expression of PRKAR1A in the fibrolamellar carcinoma negative for PRKACA rearrangements and in five control fibrolamellar carcinomas that had the classic PRKACA rearrangements by FISH. By immunohistochemistry, PRKAR1A expression was negative to weakly positive in the background liver. In the fibrolamellar carcinomas with the classic PRKACA rearrangements by FISH, PRKAR1A was strongly overexpressed, most likely in response to activation of the protein kinase A pathway. In contrast, the fibrolamellar carcinoma that lacked the PRKACA rearrangements by FISH was negative for PRKAR1A (Figures 4a and b).

The consistent loss of LFABP was not anticipated in the fibrolamellar carcinomas that co-occurred with or followed resection for hepatic adenomas, so we studied LFABP expression in a second cohort of 19 fibrolamellar carcinomas, including 11 previously published tumors ${ }^{1}$ and an additional new set of 8 tumors. The diagnosis in the additional set of eight fibrolamellar carcinomas was first confirmed at the genomic level by detection of the PRKACA rearrangement by interphase FISH: all were positive. By immunohistochemistry, all 19 cases displayed reduced or complete loss of LFABP expression, similar to the three index fibrolamellar carcinomas 

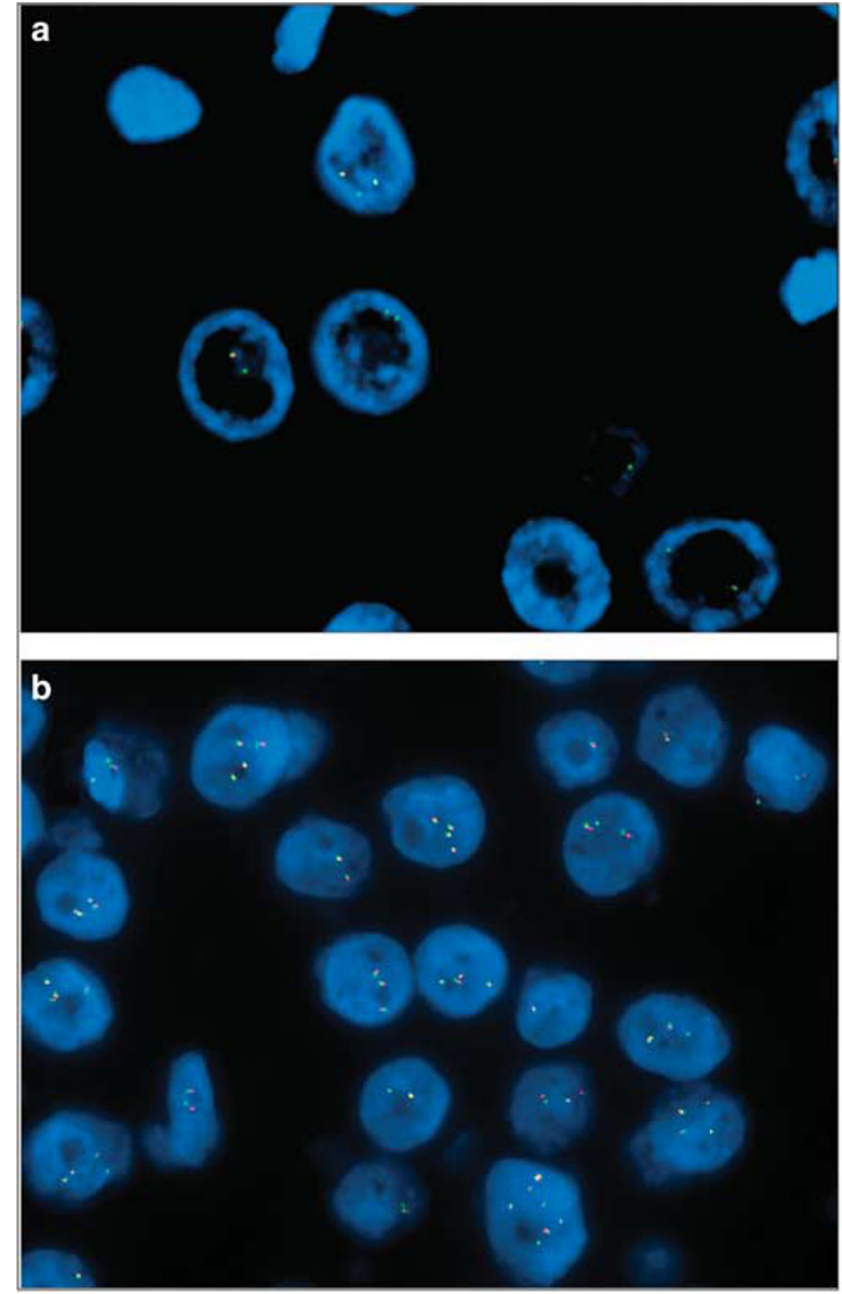

Figure 3 Fish results for PRKACA rearrangements. (a) The fibrolamellar carcinomas in each of the first three cases with adenomas all showed rearrangements of the PRKACA locus. (b) In contrast, the fibrolamellar carcinoma in the fourth case was negative for rearrangements of the PRKACA locus.

associated with hepatocellular adenomas (Table 2; Figure 5a). In fibrolamellar carcinomas that retained some LFABP expression, the tumors typically showed a mosaic pattern, with scattered individually positive tumor cells in a background of negative to very faintly positive tumor cells (Figure $5 \mathrm{~b}$ ).

To confirm the loss LFABP protein expression in fibrolamellar carcinomas, mass spectrometrybased proteomic analysis was performed on six fibrolamellar carcinomas, comparing protein levels between matched tumor and non-tumor pairs. As expected, mass spectrometry revealed overexpression of cytokeratin 7 and anterior gradient-2 (AGR2; Table 3). In addition, PRKACA was clearly overexpressed at the protein level. LFABP was 10-fold reduced in tumor cells relative to non-neoplastic cells (Table 3). As an internal control, Arginase-1 levels were similar between tumor and nonneoplastic cells.
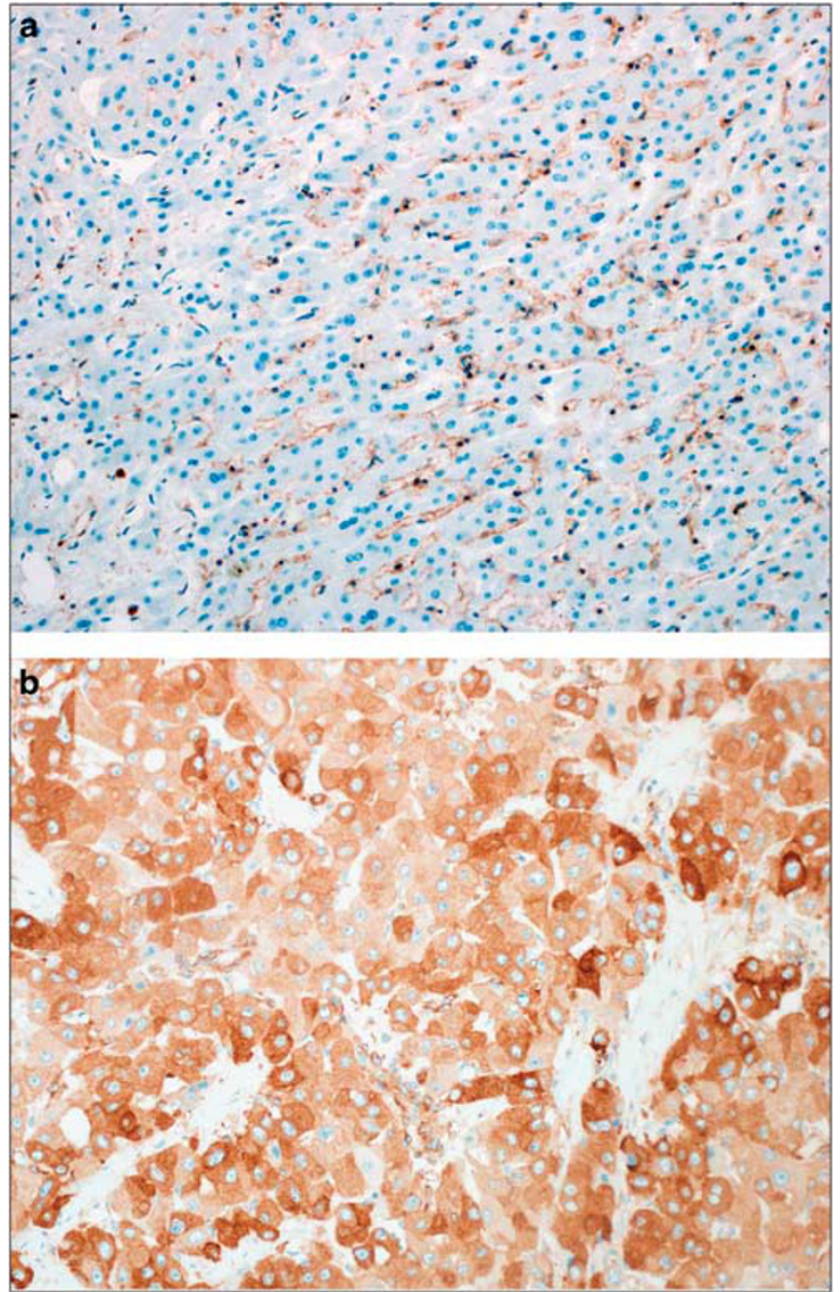

Figure 4 PRKAR1A expression in fibrolamellar carcinomas. (a) The fibrolamellar carcinoma negative for the DNAJB1PRKACA fusion shows loss of PRKAR1A expression. (b) In contrast, fibrolamellar carcinomas with the DNAJB1-PRKACA fusion show retained expression of PRKAR1A.

\section{Discussion}

Fibrolamellar carcinoma is a unique type of primary liver carcinoma that is distinct from typical hepatocellular carcinomas at the histological, immunophenotypical, and molecular levels. These unique findings are used to recognize this tumor in diagnostic surgical pathology. The morphological findings are the foundation for the diagnosis, but because of histological overlap with other tumors, the diagnosis also benefits from confirmatory tests. The combination of typical morphology and CK7 and CD68 co-expression are useful to diagnose fibrolamellar carcinomas and separate them from their mimics. ${ }^{11}$ An alternative method is detection of recurrent somatic DNAJB1-PRKACA in fibrolamellar carcinoma. $^{1}$

Although rare, cases of hepatic adenomas can develop in livers with synchronous or metachronous fibrolamellar carcinomas. This is the first series of 
Table 2 Summary of the results of LFABP immunohistochemistry on fibrolamellar carcinomas

\begin{tabular}{lcll}
\hline & $\begin{array}{c}\text { Semiquantative } \\
\text { extent of } \\
\text { LFABP-positive } \\
\text { neoplastic cells }(\%)\end{array}$ & $\begin{array}{l}\text { Intensity } \\
\text { of LFABP } \\
\text { staining }\end{array}$ & $\begin{array}{l}\text { Evaluated } \\
\text { with mass } \\
\text { spectrometry }\end{array}$ \\
\hline First index & 0 & NA & ND \\
Second index & 20 & Weak & ND \\
Third index & 10 & Weak & ND \\
1 & 10 & Moderate & ND \\
2 & 10 & Moderate & Yes \\
3 & 40 & Moderate & Yes \\
4 & 0 & NA & ND \\
5 & 0 & NA & ND \\
6 & 0 & NA & ND \\
7 & 20 & Weak & ND \\
8 & 0 & NA & ND \\
9 & 0 & NA & ND \\
10 & 0 & NA & Yes \\
11 & 0 & NA & ND \\
12 & 70 & Moderate & ND \\
13 & 5 & Weak & ND \\
14 & 30 & Weak & ND \\
15 & 20 & Weak & ND \\
16 & 0 & NA & ND \\
17 & 5 & Weak & ND \\
18 & 10 & Weak & ND \\
19 & 0 & NA & ND \\
& & & \\
\hline & & & \\
& 0 & &
\end{tabular}

Abbreviations: ND, not done; NA, not applicable.

${ }^{a}$ The other three cases of fibrolamellar carcinoma randomly chosen for mass spectrometry were not evaluated with LFABP immunohistochemistry.

such cases and shows several key findings. First, the adenomas and the fibrolamellar carcinomas are morphologically distinct: they look different histologically and are physically separated. None had transition zones. Second, none of the adenomas had a PRKACA rearrangement. Third, the hepatic adenomas were type 1 adenomas (HNF1- $\alpha$ inactivated) in three specimens, with a steatotic histology and loss of LFABP expression. Fourth, the fibrolamellar carcinomas were histologically classic, with no unusual features.

The adenoma that arose in the setting of the Carney complex had a myxoid morphology and was unclassifiable using immunohistochemistry, but showed partial LFABP loss. Interestingly, the metachronous fibrolamellar carcinoma lacked a PRKACA rearrangement by FISH and RT-PCR, but had classical morphology and CK7 and CD68 co-expression. More than $70 \%$ of families with the Carney Complex have germline mutation in PRKAR1A, a regulatory subunit of protein kinase A. Inactivation of PRKAR1A could potentially lead to excess protein kinase A signaling, providing an alternative molecular lesion that, like a PRKACA rearrangements, would lead to excess protein kinase A signaling. Although direct molecular confirmation of a PRKAR1A mutation was not possible, there was loss of PRKAR1A expression. This finding has clinical testing implications and confirms the

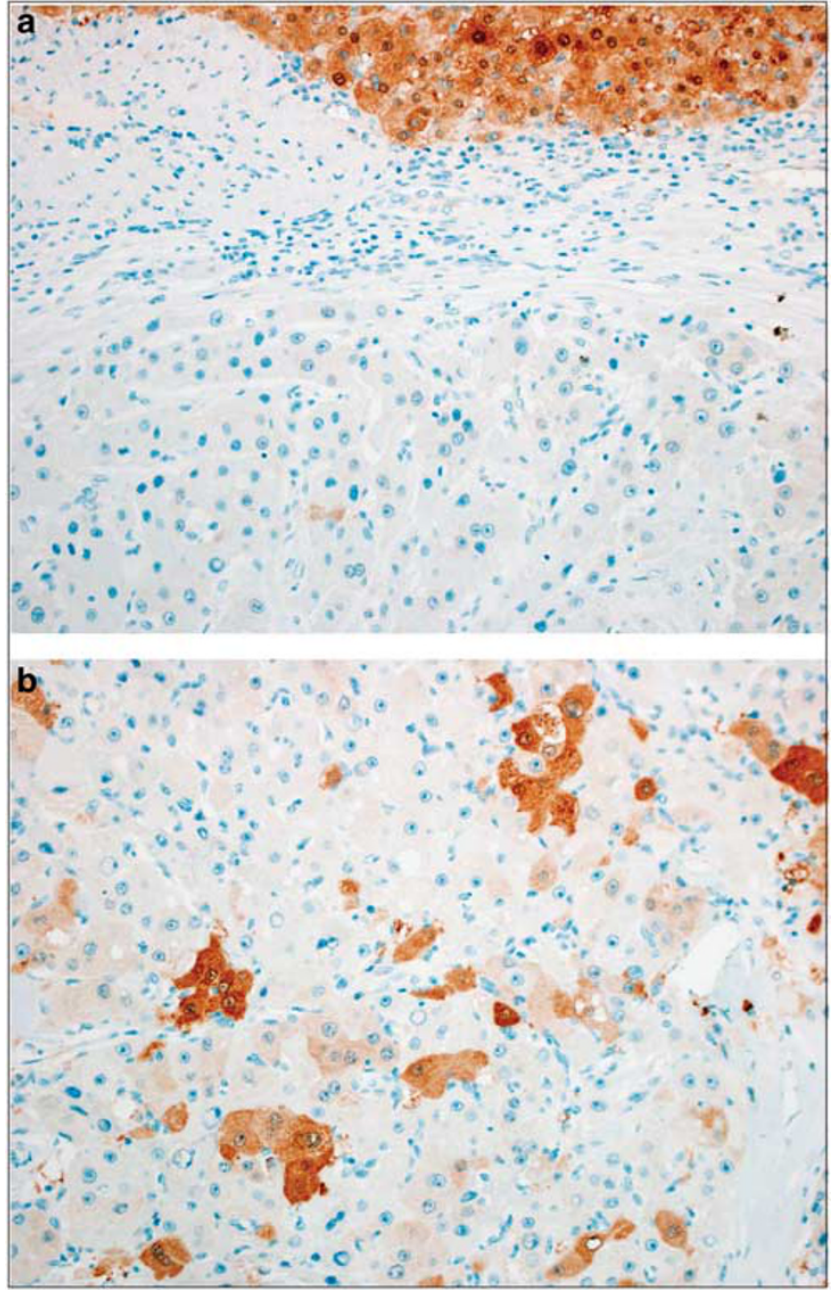

Figure 5 LFABP loss in fibrolamellar carcinomas. (a) The degree of LFABP loss varied. In most cases, the tumors showed extensive loss, with either no or residual trace staining. The non-neoplastic liver is seen at the top of the image. (b) Other cases showed a mosaic staining pattern, with scattered strongly positive tumor cells in a background of otherwise negative staining.

Table 3 The expression of various proteins in the tumor cells of fibrolamellar carcinoma compared with the matched nonneoplastic cells for six cases is shown

\begin{tabular}{lc} 
Protein & $\begin{array}{c}\text { Average fold change compared with } \\
\text { matched normal tissue }\end{array}$ \\
\hline Cytokeratin 7 & 4.4 \\
Anterior gradient-2 & $>100$ \\
PRKACA & 2.2 \\
Arginase-1 & 1.1 \\
LFABP & 0.1 \\
\hline
\end{tabular}

importance of morphology as the basis for the diagnosis of fibrolamellar carcinoma, followed by confirmatory testing.

Importantly, these data also highlight LFABP loss as a consistent finding in fibrolamellar carcinomas, 
one that was confirmed by two different methods: immunohistochemistry and mass spectrometrybased proteomics. The advantages of mass spectrometry-based proteomics are the improved quantitative capability and precision, as well as the ability to assess multiple analytes simultaneously, compared with conventional immunohistochemistry. Our data from this approach confirmed markedly reduced LFABP expression and simultaneously confirmed overexpression of other markers known to be overexpressed in fibrolamellar carcinoma; namely anterior gradient- $2,{ }^{12}$ cytokeratin 7 , and PRKACA. ${ }^{1,2}$

Taken together, these data suggest that HNF1- $\alpha$ inactivation is a key recurrent event in the biology of fibrolamellar carcinomas. LFABP is a downstream target of HNF1- $\alpha^{13}$ and bialellic loss of HNF1- $\alpha$ is associated with loss of LFABP expression, an observation that forms the foundation for using LFABP immunohistochemistry as a diagnostic tool. ${ }^{14}$ It is unknown how the loss of the tumor suppressor HNF1- $\alpha$ contributes to neoplasia, but biallelic loss of $H N F 1-\alpha$ can lead to activation of glycolysis and lipogenesis, ${ }^{15,16}$ as well as upregulation of ERBB2 and mTOR. Other possible targets include upregulation of cyclin D1 to promote cell division and upregulation of PDGFA and PDGFB, facilitating angiogenesis.

Loss of LFABP1 by immunohistochemistry is a key part of the diagnosis of type 1, or HNF1- $\alpha$ inactivated, hepatic adenomas. However, LFABP loss is not unique to type 1 hepatic adenomas. Loss of LFABP1 by immunohistochemistry is also seen in conventional hepatocellular carcinomas, ${ }^{17,18}$ in myxoid hepatic adenomas, ${ }^{10}$ and in fibrolamellar carcinomas (current study), implying that HNF1- $\alpha$ inactivation is a broadly targeted pathway in hepatocellular neoplasia. In this respect, $H N F 1-\alpha$ inactivation shares parallels with the Wnt signaling pathway, both of which are broadly targeted by both benign and malignant hepatocellular tumors.

Interestingly, $H N F 1-\alpha$ inactivation in hepatic adenomas typically results from HNF1- $\alpha$ mutations. In contrast, HNF1- $\alpha$ mutations have not been identified in genomic or transcriptomic study of fibrolamellar carcinomas. ${ }^{2,19,20}$ This suggests an alternate mechanism for the downregulation of LFABP in fibrolamellar carcinoma, perhaps through methylation or microRNAs. The mosaic pattern seen in some of the fibrolamellar carcinomas is also unusual, with scattered strongly positive tumor cells in a background of more diffuse loss of staining in tumor cells. The significance of this staining pattern is not clear, but the mosaic staining would support a possible epigenetic mechanism.

Overall, the lack of HNF1- $\alpha$ mutations in fibrolamellar carcinomas is similar to conventional hepatocellular carcinomas, where $\sim 40 \%$ of conventional hepatocellular carcinomas show loss of LFABP, but mutations are found in only $5 \%$ of cases. ${ }^{21,22}$ The diminished or absent LFABP expression in all cases of fibrolamellar carcinoma suggests $H N F 1-\alpha$ inactivation is an important molecular event in tumorgenesis and should be added to the short list of other distinctive, consistent molecular events, including AGR2 overexpression and protein kinase A activation, which results from DNAJB1-PRKACA fusion or possibly loss of PRKAR1A protein expression.

In conclusion, hepatic adenomas rarely co-occur with fibrolamellar carcinomas. Most are type 1 adenomas, sharing with fibrolamellar carcinomas the key biological event of HNF1- $\alpha$ inactivation. Our data also suggests that protein kinase A activation can rarely occur through other molecular events in fibrolamellar carcinomas. We also report the first well documented fibrolamellar carcinoma that is negative for the DNAJB1-PRKACA fusion, with the diagnosis of fibrolamellar carcinoma confirmed by histological review by liver pathologists and by co-expression of CK7 and CD68.

\section{Acknowledgments}

We are grateful for the funding support of the Department of Laboratory Medicine and Pathology Research Committee through Discretionary funds. We thank the Pathology Research Core (supported by CA 15083), Cytogenetics Core and Proteomics Core of the Mayo Clinic Medical Genome Facility for excellent technical work.

\section{Disclosure/conflict of interest}

The authors declare no conflict of interest.

\section{References}

1 Graham RP, Jin L, Knutson DL et al. DNAJB1-PRKACA is specific for fibrolamellar carcinoma. Mod Pathol 2015;28:822-829.

2 Honeyman JN, Simon EP, Robine N et al. Detection of a recurrent DNAJB1-PRKACA chimeric transcript in fibrolamellar hepatocellular carcinoma. Science 2014; 343:1010-1014.

3 Terracciano LM, Tornillo L, Avoledo $\mathrm{P}$ et al. Fibrolamellar hepatocellular carcinoma occurring 5 years after hepatocellular adenoma in a 14-year-old girl: a case report with comparative genomic hybridization analysis. Arch Pathol Lab Med 2004;128:222-226.

4 Torbenson M. Fibrolamellar carcinoma: 2012 Update. Scientifica 2012;2012:15.

5 Liu H, Sadygov RG, Yates JR 3rd. A model for random sampling and estimation of relative protein abundance in shotgun proteomics. Anal Chem 2004;76:4193-4201.

6 Vrana JA, Theis JD, Dasari S et al. Clinical diagnosis and typing of systemic amyloidosis in subcutaneous fat aspirates by mass spectrometry-based proteomics. Haematologica 2014;99:1239-1247.

7 Vrana JA, Gamez JD, Madden BJ et al. Classification of amyloidosis by laser microdissection and mass spectrometry-based proteomic analysis in clinical biopsy specimens. Blood 2009;114:4957-4959. 
8 Tabb DL, Fernando CG, Chambers MC. MyriMatch: highly accurate tandem mass spectral peptide identification by multivariate hypergeometric analysis. J Proteome Res 2007;6:654-661.

9 Ma ZQ, Dasari S, Chambers MC et al. IDPicker 2.0: improved protein assembly with high discrimination peptide identification filtering. J Proteome Res 2009;8: 3872-3881.

10 Salaria SN, Graham RP, Aishima S et al. Primary hepatic tumors with myxoid change: morphologically unique hepatic adenomas and hepatocellular carcinomas. Am J Surg Pathol 2015;39:318-324.

11 Ross HM, Daniel HD, Vivekanandan P et al. Fibrolamellar carcinomas are positive for CD68. Mod Pathol 2011;24:390-395.

12 Vivekanandan P, Micchelli ST, Torbenson M. Anterior gradient-2 is overexpressed by fibrolamellar carcinomas. Hum Pathol 2009;40:293-299.

13 Akiyama TE, Ward JM, Gonzalez FJ. Regulation of the liver fatty acid-binding protein gene by hepatocyte nuclear factor 1alpha (HNF1alpha). Alterations in fatty acid homeostasis in HNF1alpha-deficient mice. J Biol Chem 2000;275:27117-27122.

14 Bioulac-Sage P, Rebouissou S, Thomas C et al. Hepatocellular adenoma subtype classification using molecular markers and immunohistochemistry. Hepatology 2007;46:740-748.

15 Pelletier L, Rebouissou S, Paris A et al. Loss of hepatocyte nuclear factor 1alpha function in human hepatocellular adenomas leads to aberrant activation of signaling pathways involved in tumorigenesis. Hepatology 2010;51:557-566.

16 Rebouissou S, Imbeaud S, Balabaud C et al. HNF1alpha inactivation promotes lipogenesis in human hepatocellular adenoma independently of SREBP-1 and carbohydrate-response element-binding protein (ChREBP) activation. J Biol Chem 2007;282:14437-14446.

17 Inoue M, Takahashi Y, Fujii T et al. Significance of downregulation of liver fatty acid-binding protein in hepatocellular carcinoma. World J Gastroenterol 2014;20:17541-17551.

18 Wang B, Tao X, Huang CZ et al. Decreased expression of liver-type fatty acid-binding protein is associated with poor prognosis in hepatocellular carcinoma. Hepatogastroenterology 2014;61:1321-1326.

$19 \mathrm{Xu}$ L, Hazard FK, Zmoos AF et al. Genomic analysis of fibrolamellar hepatocellular carcinoma. Hum Mol Genet 2015;24:50-63.

20 Darcy DG, Chiaroni-Clarke R, Murphy JM et al. The genomic landscape of fibrolamellar hepatocellular carcinoma: whole genome sequencing of ten patients. Oncotarget 2015;6:755-770.

21 Schulze K, Imbeaud S, Letouze E et al. Exome sequencing of hepatocellular carcinomas identifies new mutational signatures and potential therapeutic targets. Nat Genet 2015;47:505-511.

22 Zucman-Rossi J, Villanueva A, Nault JC et al. The genetic landscape and biomarkers of hepatocellular carcinoma. Gastroenterology 2015;149:1226-1239. 